\title{
BAS-ELM based UAV hyperspectral remote sensing inversion modeling of rice canopy nitrogen content
}

\author{
Fenghua $\mathrm{Yu}^{1,2}$, Shuai Feng ${ }^{1}$, Weixiang $\mathrm{Yao}^{1,2}$, Dingkang Wang ${ }^{1}$, Simin $\mathrm{Xing}^{1}$, Tongyu $\mathrm{Xu}^{1,2^{*}}$ \\ (1. College of Information and Electrical Engineering, Shenyang Agricultural University, Shenyang 110866, China; \\ 2. Liaoning Agricultural Information Engineering Technology Research Center, Shenyang 110866, China)
}

\begin{abstract}
The rapid, nondestructive, and accurate estimation of nitrogen content in rice can help to obtain the growth condition of rice in time, which is of great significance for guiding rice field management. In order to improve the accuracy of high-spectral inversion of rice canopy nitrogen content, the discrete wavelet multiscale decomposition (DWMD) was used to downscale the high-spectral information in the range of $400 \mathrm{~nm}$ to $1000 \mathrm{~nm}$ by using the UAV hyperspectral image data and the synchronously measured rice canopy nitrogen content as the data source, from which the hyperspectral characteristic variables for rice nitrogen content inversion modeling were extracted. And using the dimensionality reduction variables as the data base, three neural network inversion methods, including extreme learning machines (ELM), particle swarm optimization for extreme learning machines (PSO-ELM), and beetle antennae search algorithm for extreme learning machines (BAS-ELM), were used to establish the rice nitrogen content drone hyperspectral remote sensing inversion model, and the results showed that: (1) The hyperspectral range from $400 \mathrm{~nm}$ to $1000 \mathrm{~nm}$ was dimensionally reduced by DWMD, and finally the continuous hyperspectral reflectance information was dimensionally reduced to sixteen discrete hyperspectral features for subsequent inversion modelling of rice nitrogen content.(2) In the model adopted in this study, BAS-ELM has the highest accuracy, where the $R^{2}$ of training data, the $R^{2}$ of test data, the RMSE of training data, and the RMSE of test data is were 0.864, 0.863, 0.247, 0.254.
\end{abstract}

Keywords: UAV, Nitrogen inversion, rice, Hyperspectral dimensionality reduction, ELM DOI: $10.33440 /$ j.ijpaa.20200303.105

Citation: Yu F H, Feng S, Yao W X, Wang D K, Xing S M, Xu T Y. BAS-ELM based UAV hyperspectral remote sensing inversion modeling of rice canopy nitrogen content. Int J Precis Agric Aviat, 2020; 3(3): 59-64.

\section{Introduction}

Rice is one of the most important food crops in the world, and rice production is of great significance to food security of the world ${ }^{[1]}$. To increase rice yield, the managers should apply chemical fertilizers during rice production ${ }^{[2,3]}$. The application of nitrogen fertilizer is a key factor to ensure the final yield of rice. In addition, rice yield is also closely related to photosynthesis ${ }^{[4]}$. Relevant studies have shown that rice photosynthesis is related to nitrogen, and rice leaf photosynthetic rate is highly correlated with nitrogen content, to reach a significant level. When the nitrogen content is scarce during rice growth, the chlorophyll content of the leaves will decrease, the content and activity of enzymes related to photosynthesis will decrease, and the total leaf area will decrease, which will eventually lead to a decrease in dry matter accumulation ${ }^{[5]}$; but Excessive application of nitrogen fertilizer will also lead to reduced rice yields and ultimately reduced yields ${ }^{[6,7]}$. This will not only increase crop planting costs, but also have adverse effects on the ecological environment, such as soil

Received data: 2020-07-03 Accepted data:2020-09-18

Biographies: Fenghua Yu, $\mathrm{PhD}$, research interests: agriculture remote sensing, Email: adan@syau.edu.cn; Shuai Feng, PhD, research interests: agriculture remote sensing, Email: int_crazy@163.com; Weixiang Yao, PhD, research interests: Precision agricultural aviation, Email: 1913835329@qq.com; Dingkang Wang, Master student, research interests: agriculture remote sensing, Email: Wangdingkang1997@163.com; Simin Xing, Master student, research interests: agriculture remote sensing, Email: 953982392 @qq.com;

* Corresponding author: Tongyu Xu, PhD, Professor, research interests: Precision agricultural aviation, Mailing Address: College of Information and Electrical Engineering, Shenyang Agricultural University, Shenyang, China. Email: xutongyu@syau.edu.cn. compaction and eutrophication of water bodies ${ }^{[8]}$. Some studies have pointed out that the amount of nitrogen fertilizer used in the current rice production management process is too high, which exceeds the optimal amount of nitrogen fertilizer required for high rice yield, and the phenomenon of excessive application of chemical fertilizers is more serious ${ }^{[9-11]}$. Therefore, the rapid detection and evaluation of nitrogen content during rice growth is an important prerequisite for precise rice fertilization management according to demand.

At present, the method of rice nitrogen diagnosis is generally to take destructive rice field samples and conduct chemical analysis of rice tissues indoors. Although the detection results obtained by indoor chemical analysis are more accurate, this method requires a lot of human labor and material resources, which is time-consuming and has a certain time $\operatorname{lag}^{[12]}$. Therefore, the use of UAV hyperspectral technology to diagnose the nitrogen content of rice can make up for the shortcomings of the aforementioned traditional methods ${ }^{[13]}$. The UAV low-altitude remote sensing platform is a fast-developing near-Earth remote sensing method in recent years ${ }^{[14]}$. Its platform has high flexibility, rich data acquisition. Compared with other remote sensing methods, it has certain unique advantages ${ }^{[15]}$. The remote sensing method of UAV hyperspectral remote sensing inversion and estimation of the nitrogen content in the rice canopy at the near-earth scale has important practical significance for assisting the precise fertilization and pesticide application in the field.

Existing studies have shown that when the nitrogen content of rice changes, it will cause changes in the reflectance of different wavelengths at the spectral level. Because hyperspectral information has a relatively high data dimension, it is usually necessary to reduce the dimension of hyperspectral data first, and 
then establish a quantitative inversion model with rice nitrogen content ${ }^{[16]}$. Relevant researchers have made certain research results in using UAV hyperspectral technology to estimate the nitrogen content of rice. He et al. ${ }^{[17]}$ established a vertical distribution model of the relative canopy height. These results provide technical support for the rapid, accurate, and non-destructive identification of the vertical distribution of nitrogen in rice canopies. Jay et al. ${ }^{[18]}$ exploited the centimeter resolution of UAV multispectral imagery to inverse the nitrogen content in sugar beet crops. Zhang et al. ${ }^{[19]}$ studied 12 spectral indices combined with partial least square analysis. This approach was applied for estimating chlorophyll content of rice leaves from plants subjected to different nitrogen levels, and a root mean square error of cross-validation of 0.506 , a coefficient of determination of $97.8 \%$ and a ratio of performance to deviation of 4.6 for all rice varieties indicated this as a preferable procedure. This study demonstrates that Vis/NIR spectroscopy can have a great potential for identification of rice varieties and evaluation of nitrogen fertilizer levels. Camino et al. ${ }^{[20]}$ improved nitrogen retrievals with airborne-derived fluorescence and plant traits quantified from hyperspectral imagery in the context of precision agriculture. In the work of Stavrakoudis et al. ${ }^{[21]}$, the reflectance values and vegetation indices obtained from a compact multispectral sensor onboard an unmanned aerial vehicle were used as inputs. At the booting stage, similarly high accuracies were achieved for yield, $\mathrm{N}$ concentration, $\mathrm{N}$ uptake, biomass, and plant height, using inputs from either two or three images. The results of the present study can be useful for providing $\mathrm{N}$ recommendations for the two top-dressing fertilizations in rice cultivation, through a cost-efficient workflow. Masemola et al. ${ }^{[22]}$ analyzed the leaf structure interference factors affecting the estimation of leaf nitrogen content, and constructed a characteristic wavelength selection method with $R^{2}$ of 0.82 and RMSE of 0.13 , besides determined typical nitrogen bands of crop leaves, which can effectively monitor Nitrogen content of leaves during different periods. Klem et al. ${ }^{[23]}$ studied the special and thermal indices which can provide satisfactory estimations of perspective of interactions between water and $\mathrm{N}$ discount, grain yield, $\mathrm{N}$ uptake, and mathematical responses.

At present, in the research of rice nitrogen content retrieval from UAV low altitude remote sensing, most of the feature bands are selected from multi-dimensional Hyperspectral Information, and the statistical regression model between vegetation index and rice nitrogen content is established by using the characteristic band to build vegetation index ${ }^{[24-26]}$. This method has ideal retrieval effect for specific varieties in specific areas, but the model is still insufficient in generality. The previous work of using hyperspectral analysis technology to detect nitrogen content mainly focused on two aspects: establishing various vegetation indices, using multiple linear or non-linear regression method to establish the inversion model between the index and nitrogen content; or modeling all bands of hyperspectral data of rice canopy by PCA, PLS and other $\operatorname{methods}^{[27,28]}$

The purpose of this study was to study the inversion of nitrogen content in the rice canopy and leaf layer in the cold region of Northeast China, so as to solve the problem of rapid, accurate and nondestructive diagnosis of nutrition status in rice growth process and to improve the accuracy of nitrogen content inversion. In order to realize the accurate inversion of rice canopy chlorophyll content in the cold region of northeast China, this study used UAV hyperspectral remote sensing platform to obtain the hyperspectral image data of the key growth period of rice canopy, and used the discrete wavelet multiscale decomposition (DWMD) to hyperspectral features extraction for the response of rice nitrogen content, then established the limit learning machine inversion model based on the improved beetle antennae search algorithm (BAS-ELM).

\section{Materials and methods}

\subsection{Study area and design}

This study was carried out in the pilot field of precision agriculture aviation team of Shenyang agricultural university. The pilot place was in Liutiao Village, Shenyang City, Liaoning Province $\left(123^{\circ} 63^{\prime} \mathrm{E}, 42^{\circ} 01^{\prime} \mathrm{N}\right)$, and the experiment was carried out from June to August 2020. The experimental variety of rice was "gengyou653" and the reproductive stages of rice covered in this study include rejuvenation, tillering, and pulling stage. The data acquisition activity depended on the weather condition, which avoided the rainy and windy circumstances. The test plot was designed as four nitrogen fertilizer gradient treatments (Figure 1), namely $\mathrm{CK}, \mathrm{N} 1, \mathrm{~N} 2$ and N3, with ridges separating the plots. CK was the control group, i.e. no basal fertilizer was applied; N1 was the local standard nitrogen basal fertilizer application level of $45 \mathrm{~kg} / \mathrm{hm}^{2}$; N2 was the low nitrogen fertilizer application level of $22.5 \mathrm{~kg} / \mathrm{hm}^{2}$; N3 was the high nitrogen fertilizer application level of $67.5 \mathrm{~kg} / \mathrm{hm}^{2}$; phosphorus and potassium fertilizer were applied according to the local standard application level, of which the standard phosphorus application level of $45 \mathrm{~kg} / \mathrm{hm}^{2}$ was applied. $51.75 \mathrm{~kg} / \mathrm{hm}^{2}$ and the standard application rate of potash is $18 \mathrm{~kg} / \mathrm{hm}^{2}$. Other field management is carried out according to the normal local level.

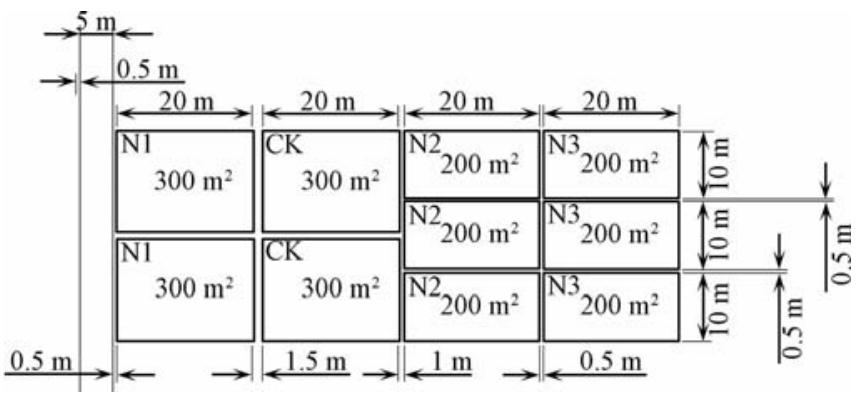

Note: $\mathrm{CK}$ is the control group; the application rate of $\mathrm{N} 1$ is $45 \mathrm{~kg} \cdot \mathrm{hm}^{-2}$, that of $\mathrm{N} 2$ is $22.5 \mathrm{~kg} \cdot \mathrm{hm}^{-2}$, and that of $\mathrm{N} 3$ is $67.5 \mathrm{~kg} \cdot \mathrm{hm}^{-2}$.

Figure 1 Test site of this study

\subsection{UAV hyperspectral remote sensing image acquisition}

The UAV hyperspectral platform adopts the M600 PRO six-rotor UAV from DJI Co., Ltd. and the hyperspectral sensor uses the GaiaSky-mini that a built-in push-scan airborne hyper-spectral imaging system from Sichuan Shuang li Hepu Company (Sichuan, China). The hyperspectral band range is $400-1000 \mathrm{~nm}$, the resolution is $3.5 \mathrm{~nm}$, and the number of effective bands is 170 .

The data acquisition time of hyperspectral remote sensing platform of UAV is between 11:00-12:00 a.m. in each test. During each flight, the period with relatively stable solar light intensity was selected, and the flight height of UAV is $50 \mathrm{~m}$. In this study, the DN value of rice canopy was transformed into the hyperspectral reflectance information of rice canopy by field calibration. A black and white calibration blanket was placed on the path in the rice field. The hyperspectral information of the calibration blanket is included in the hyperspectral image collected by the UAV during the hyperspectral acquisition process. The DN value is converted into the spectral reflectance by formula (1). 


$$
\rho_{t}=\frac{D N_{t}-D N_{1}}{D N_{2}-D N_{1}}\left(\rho_{2}-\rho_{1}\right)+\rho_{1}
$$

where, $\rho_{t}$ and $D N_{t}$ are the reflectance and $\mathrm{DN}$ values of the ground object to be converted, and the reflectance of the calibration blanket are $\rho_{1}$ and $\rho_{2}$, respectively; $D N_{1}$ and $D N_{2}$ are the DN values of the calibration blanket.

We used the ENVI5.4 software to extract the hyperspectral data of the acquired hyperspectral remote sensing image, firstly using the K-means method is used for hyperspectral data classification. Since the rice field is mainly composed of rice, water, soil and other features, and taking into account factors such as classification errors, the number of categories is set at twice the number of major features and the maximum number of categories is set to 6 in this study. After that, the average spectrum of each area of interest was calculated as hyperspectral information for each test plot.

\subsection{Measurement of nitrogen content}

Rice was destructively sampled in each experimental plot and brought back to the laboratory, and four holes of rice were collected from each plot and all fresh leaves of these rice were cut and placed in an oven at $105^{\circ} \mathrm{C}$ for $30 \mathrm{~min}$ to be dried at $65^{\circ} \mathrm{C}$ to a co Normalization is taken as nstant volume. After weighing, they were crushed and the ground powder was put into labeled self-sealing bags for the determination of the nitrogen content $(\mathrm{N}$, $\mathrm{mg} / \mathrm{g}$ ) of the leaves using the traditional Kjeldahl method.

\subsection{UAV hyperspectral dimensionality reduction methods}

The full-band spectra acquired by the unmanned hyperspectral remote sensing system contain a large amount of redundant information unrelated to the nitrogen content of rice, which can lead to increased model error during inversion model building. Therefore, extracting useful information from the hyperspectral data is a prerequisite for building robust and accurate models. In this study, the discrete wavelet multiscale decomposition (DWMD) was used to extract hyperspectral information, which was then used as the input variable of the nitrogen content inversion model.

The DWMD can accurately decompose the spectral signal in the time domain and frequency. In the domain for leaf spectral information, the transformation of the signal in the time domain is equivalent to the transformation of the spectral data in the spectral bands, so the wavelet basis function can be expressed as a degree decomposition:

$$
\phi_{a, b}(\lambda)=\frac{1}{\sqrt{a}} \phi\left(\frac{\lambda-b}{a}\right) a, b \in R ; a>0 ; \int_{-\infty}^{+\infty} \phi(\lambda) d \lambda=0
$$

where, $a$ is the telescoping factor; $b$ is the panning factor; $\lambda$ is an independent variable, and the function mean is 0 . The discrete wavelet transform is the discrete of the decomposition scale and panning, and it is a one-dimensional input signal. The discrete wavelet transform coefficient is the approximation of the base function to the signal after discrete scaling and panning, and can be expressed by Equation (3):

$$
W_{j, k}=\left(f(\lambda), \phi_{j, k}(\lambda)\right)
$$

where the wavelet function can be calculated by Equation (4):

$$
\phi_{j, k}(\lambda)=2^{\frac{-j}{2}} \phi\left(2^{-j} \lambda-K\right)
$$

where, $j$ and $k$ are the $j$-th decomposition and $k$-th wavelet coefficients, respectively, and the scale of discrete wavelet variation is usually taken as a binary sequence, $j=2,4,8, \ldots$, to make the calculation more efficient. In the multi-scale signal decomposition based on discrete wavelet transform algorithm, the decomposed wavelet coefficients are the approximate coefficients for recording low frequency signals and the detail coefficients for recording high frequency detail signals. Coefficients of wavelet approximation were selected as inputs for the inverse model.

\subsection{BAS-ELM inversion modeling of nitrogen content}

In this study, the extreme learning machine model based on beetle antennae search algorithm (BAS) was used to retrieve the nitrogen content of rice canopy. BAS is an intelligent algorithm proposed in 2017 based on the principle of sky cow foraging, for multi-objective function optimization. Since rice nitrogen content drone hyperspectral remote sensing inversion model belongs to the multi-objective function model, the BAS approach is suitable for this study. BAS is based on the principle that the beetle's left and right antennae can sense the intensity of the odor emitted by food. BAS is based on the information perception of a single beetle, which updates its flight direction by knowing its own local information. Therefore, BAS has the characteristics of being computationally simple and flexible. Therefore, in this paper, we will study the optimization of the model parameters of an extreme learning machine based on BAS (Figure 2).

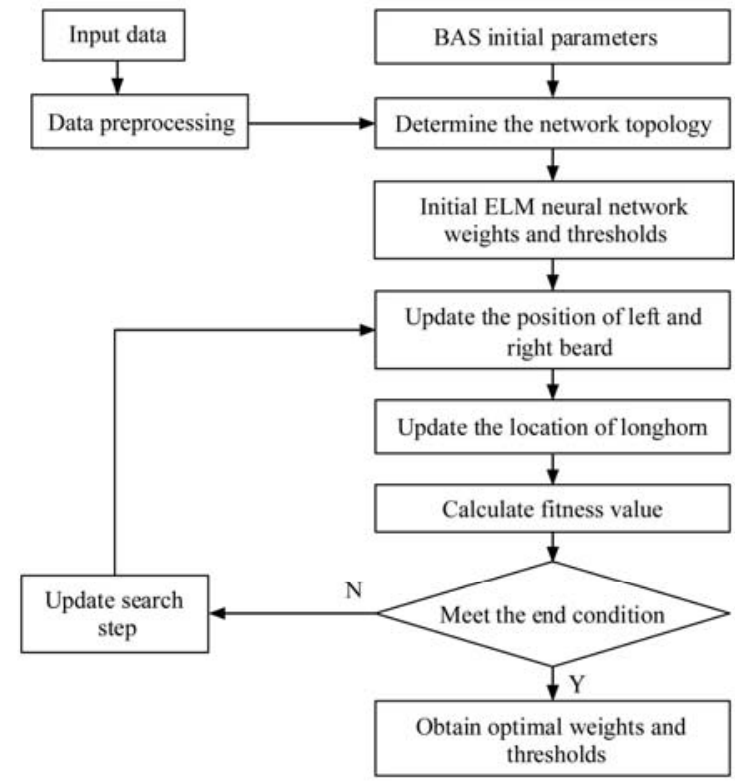

Figure 2 BAS-ELM method

The BAS algorithm flow consists of two stages:

Step 1: For an optimization problem in an n-dimensional space, we use $x l$ to represent the left whisker coordinates, $x r$ to represent the right whisker coordinates, $x$ to represent the centroid coordinates, and $d 0$ to represent the distance between the two whiskers. According to hypothesis 7, the head of the beetle is arbitrarily oriented, so the direction of the vector from the right beard to the left beard of the beetle is also arbitrary, so a random vector $\operatorname{dir}=\operatorname{rands}(n, 1)$ can be generated to represent it. Normalization is taken as: dir=dir/norm(dir); we can get $x l-x r=$ $d 0 * d i r$ in this way; obviously, $x l, x r$ can also be expressed as an expression for the center of mass:

$$
\begin{aligned}
& x l=x+d 0 * \operatorname{dir} / 2 \\
& x r=x-d 0 * \operatorname{dir} / 2
\end{aligned}
$$

Step 2: For the value function $\mathrm{f}$ to be optimized, find the values of the left and right whiskers: fleft $=f(x l)$; fright $=f(x r)$; Thus the values of two magnitudes can be determined:

- If fleft $<$ fright, in order to find the minimum value of $f$, the long-horned beetle travels to the left direction step by step, that is:

$$
x=x+\text { step } * \text { normal }(x l-x r)
$$

- If fleft $>$ fright, in order to find the minimum value of $f$, the 
long-horned beetle travels to the right direction step by step, that is:

$$
x=x-\operatorname{step} * \operatorname{normal}(x l-x r)
$$

- The above two cases can be written uniformly using the symbolic function sign:

$$
\begin{aligned}
x & =x-\text { step } * \text { normal }(x l-x r) * \operatorname{sign}(f l e f t-f r i g h t) \\
& =x-\text { step } * \operatorname{dir} * \operatorname{sign}(\text { fleft }- \text { fright })
\end{aligned}
$$

In this study, the RMSE and coefficient of determination $\left(R^{2}\right)$ were used as evaluation criteria for assessing the accuracy of the UAV hyperspectral remote sensing inversion of rice canopy nitrogen content in cold area.

\section{Results and discussion}

\subsection{Data processing}

A total of 260 sets test data samples were collected in this study, and the samples were divided according to the 3:1 ratio of training set and validation set according to the Kennard-Stone algorithm, 195 of which were randomly selected as the modeling data set and the other 65 sets as the validation data set. The maximum nitrogen content of the sample set was $4.874 \mathrm{mg} / \mathrm{g}$ and the minimum nitrogen content was $1.060 \mathrm{mg} / \mathrm{g}$, with a coefficient of variation of 0.32 .

\subsection{Results of hyperspectral dimensionality reduction}

The determination of the wavelet master function and the best decomposition scale is one of the key aspects of the wavelet transform for feature extraction. With the discrete wavelet transform performed on multiple scales, the wavelet master function and the decomposition scale can be considered as the best choice if the decomposed wavelet information can both reflect the contour characteristics of the spectrum and effectively compress the data.

The discrete wavelet transform is performed on the $\mathrm{UAV}$-acquired hyperspectral using db10, coif5, and sym8 wavelet master functions on the $(j=1,2, \ldots, 12)$ scale, respectively, and is recorded as scale 1 to 12 (Level 1-12). After the discrete wavelet multiscale decomposition on the hyperspectral data, the number of approximation coefficients obtained from each level of grading is extracted, and the compression rate with the decomposition level is obtained, as shown in Figure 3. The wavelet approximation signal characterizes the profile of the spectrum, and the signal reconstruction is performed on the approximation coefficients of each layer under different wavelet master functions to calculate the correlation coefficients between each reconstructed spectral signal and the original spectral signal, as shown in Figure 4 and Table 1.

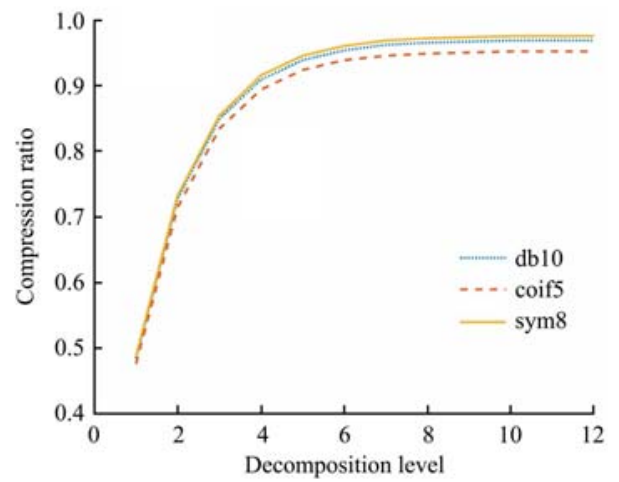

Figure 3 Compressibility for different wavelet functions

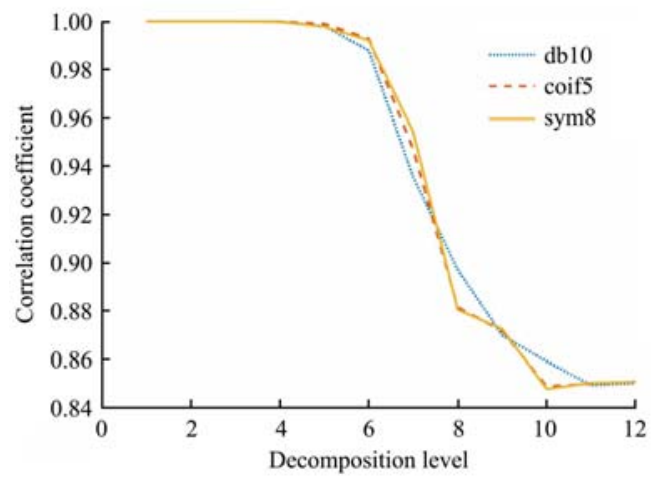

\begin{tabular}{|c|c|c|c|c|c|c|c|c|c|}
\hline \multirow{2}{*}{$\begin{array}{l}\text { Decomposition } \\
\text { level }\end{array}$} & \multicolumn{3}{|c|}{$\mathrm{db} 10$} & \multicolumn{3}{|c|}{ coif5 } & \multicolumn{3}{|c|}{ sym8 } \\
\hline & Relevance $t$ & $\begin{array}{c}\text { Compression } \\
\text { ratio }\end{array}$ & $\begin{array}{l}\text { Approximate } \\
\text { number }\end{array}$ & Relevance $t$ & $\begin{array}{c}\text { Compression } \\
\text { ratio }\end{array}$ & $\begin{array}{l}\text { Approximate } \\
\text { number }\end{array}$ & Relevance $t$ & $\begin{array}{c}\text { Compression } \\
\text { ratio }\end{array}$ & $\begin{array}{l}\text { Approximate } \\
\text { number }\end{array}$ \\
\hline 1 & 1.00 & 0.48 & 310 & 1.00 & 0.48 & 315 & 1.00 & 0.49 & 308 \\
\hline 2 & 1.00 & 0.73 & 164 & 1.00 & 0.71 & 172 & 1.00 & 0.73 & 161 \\
\hline 3 & 1.00 & 0.85 & 91 & 1.00 & 0.83 & 100 & 1.00 & 0.85 & 88 \\
\hline 5 & 1.00 & 0.94 & 37 & 1.00 & 0.92 & 46 & 1.00 & 0.95 & 33 \\
\hline 6 & 0.99 & 0.95 & 28 & 0.99 & 0.94 & 37 & 0.99 & 0.96 & 24 \\
\hline 7 & 0.94 & 0.96 & 23 & 0.95 & 0.95 & 33 & 0.95 & 0.97 & 19 \\
\hline 8 & 0.90 & 0.97 & 21 & 0.88 & 0.95 & 31 & 0.88 & 0.97 & 17 \\
\hline 9 & 0.87 & 0.97 & 20 & 0.87 & 0.95 & 30 & 0.87 & 0.97 & 16 \\
\hline 10 & 0.86 & 0.97 & 19 & 0.85 & 0.95 & 29 & 0.85 & 0.98 & 15 \\
\hline 12 & 0.85 & 0.97 & 19 & 0.85 & 0.95 & 29 & 0.85 & 0.98 & 15 \\
\hline
\end{tabular}

Figure 4 Correlation coefficients for different wavelet functions

Table 1 Number of decompositions under different wavelet generating functions

From Table 2, the number of approximation coefficients eventually stabilizes when the number of decomposition layers reaches 10. Compared with the other two types of master functions, coif5 wavelet approximation coefficient number, the data compression capacity of the weakest, which sym8 wavelet master function of the data compression capacity of the strongest. It can be seen from Figure 2 and Figure 3 that, using the db10 wavelet master function combined with the decomposition layers
$7-12$, the correlation coefficient change is more consistent compared with the other two types of wavelet master functions. From the table, after the decomposition of the 9th layer, sym8 wavelet function has the least number of approximate coefficients and the highest correlation coefficient. Therefore, considering the data compression and the ability to preserve the original spectrum, the sym 8 wavelet function is the most effective when decomposed in the 9 th layer. 
Table 2 Results of three modeling methods by ELM, PSO-ELM, BAS-ELM

\begin{tabular}{cccc}
\hline Methods & ELM & PSO-ELM & BAS-ELM \\
\hline \multirow{2}{*}{$R^{2}$} & 0.733 (train) & 0.787 (train) & 0.864 (train) \\
& 0.740 (test) & 0.812 (test) & 0.863 (test) \\
\hline \multirow{2}{*}{ RMSE } & 0.369 (train) & 0.333 (train) & 0.247 (train) \\
& 0.382 (test) & 0.339 (test) & 0.254 (test) \\
\hline
\end{tabular}

For the discrete wavelet transform, the low-frequency approximation coefficients reflect the distinct absorption characteristics of the original spectrum and determine the shape of the entire hyperspectral, so the decomposed wavelet approximation coefficients are used as inputs to the model.

\subsection{Inversion results of BAS-ELM for nitrogen content}

In this study, extreme learning machines (ELM), Particle swarm optimization for extreme learning machines (PSO-ELM), and beetle antennae search algorithm for extreme learning machines (BAS-ELM), were used to develop an inversion model of rice nitrogen content using the results of DWMD downscaling as input and rice nitrogen content as output.

PSO and BAS were used to optimize the weight and threshold of ELM, and $R^{2}$ was selected as the fitness function to evaluate the nitrogen content model. The PSO optimum values of the parameters are pop $=50, \mathrm{w}=0.9 \sim 0.3, \mathrm{C} 1=1.25, \mathrm{C} 2=1.25$, and $\mathrm{m}=0.2$. To test the prediction accuracy and learning speed of ELM model, the maximum number of iterations of model training is set as 300. $R^{2}$ and RMSE performance indexes of model training samples and test samples are selected to compare and analyze the models established by the three methods. The results are shown in Table 2.

From Table 2, we can see that BAS-ELM is the most effective of the three models, with an $R^{2}$ of 0.864 and an RMSE of 0.247 for the training set, indicating that the accuracy and generalization ability of the model are satisfactory.

The model accuracy of the rice nitrogen content inversion model using the traditional limit learning machine algorithm is weaker than that of the rice nitrogen content inversion model using the particle swarm algorithm and beetle antennae search algorithm. This result is mainly due to the fact that the model parameters of the ELM alone are set at the time of modeling and lack the optimization process, i.e., it is not possible to determine whether the given parameters are optimal solutions. Instead, the parameters of the ELM model are continuously optimized iteratively through the multi-objective function optimization algorithm, with the error size between the calculated value and the true value as the measurement basis, and the ELM model parameters are determined by setting the error threshold, thus improving the accuracy of rice nitrogen content inversion.

\subsection{Accuracy analysis of nitrogen content inversion model}

From the three nitrogen content inversion models, the performance of BAS-ELM is significantly better than ELM and PSO-ELM. However, the number of individuals in the BAS algorithm is only set to one, thus the search space range is limited, and there is a possibility of falling into the local optimal solution when solving complex problems. It is in principle the same as other groupwise algorithms, which is the common problem of a statistical optimization algorithm. Compared with other optimization algorithms, BAS is simple and easy to use in terms of model structure and parameter setting, which has attracted the attention of many researchers. The inversion accuracy of nitrogen content established by the three methods is shown in Figure 5. In the model adopted in this study, BAS-ELM has the highest accuracy, where the R2 of training data, the R2 of test data, the RMSE of training data, and the RMSE of test data is were 0.864 , $0.863,0.247,0.254$.
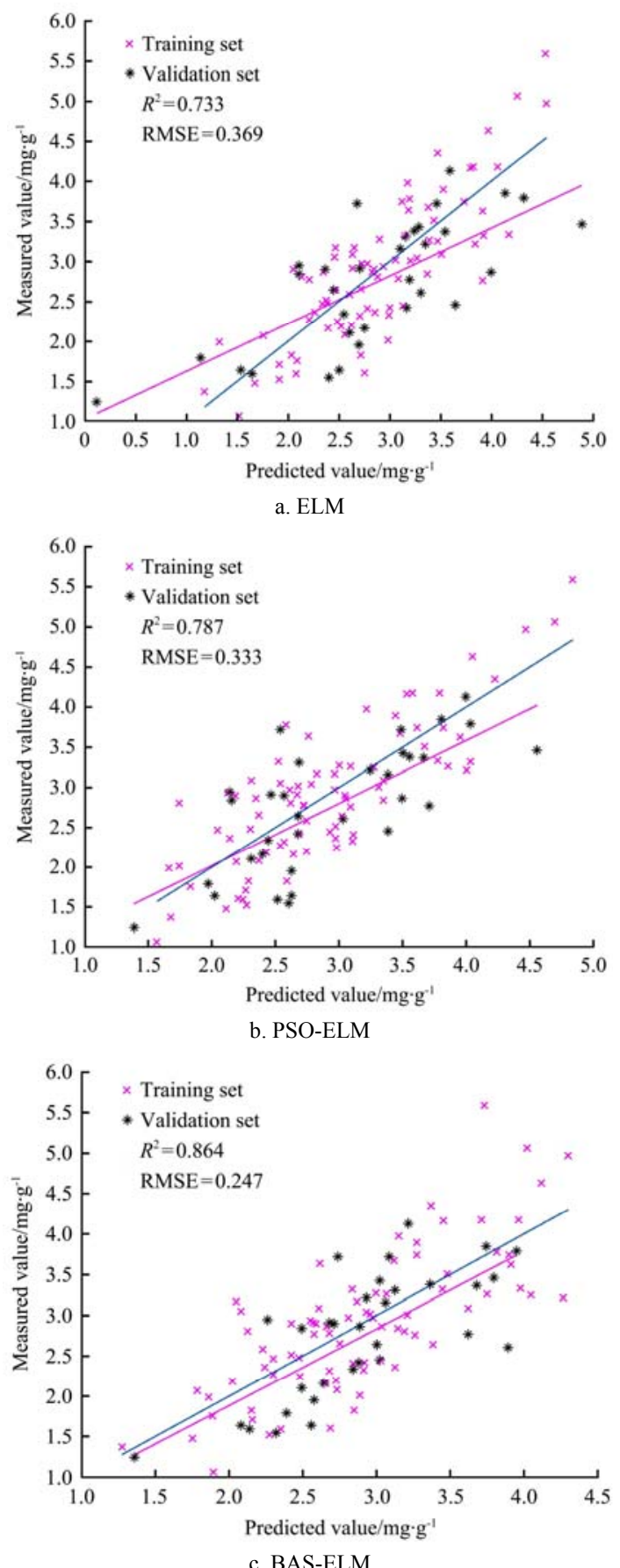

Figure 5 Results of rice nitrogen content inversion

From the inversion results, the extreme learning machine inversion model of rice nitrogen content using the micro-particle swarm optimization algorithm and the bull whisker optimization algorithm is significantly better than the traditional extreme learning machine model. This is because with the continuous iterative optimization on the ELM model parameters, the inversion model is capable to seek for better parameters with smaller errors. As a contrast, the traditional ELM model parameters are mostly given empirically and cannot achieve the optimal model effect.

\section{Conclusions}

This paper is based on the hyperspectral remote sensing image 
data of rice unmanned aerial vehicle (UAV) in Shenyang, Liaoning, China, while using destructive sampling to obtain rice nitrogen content, and the hyperspectral remote sensing image is downscaled by the discrete wavelet multiscale decomposition (DWMD) to extract rice hyperspectral remote sensing features. On this basis, ELM, PSO-ELM, and BAS-ELM, were used to establish the hyperspectral remote sensing inversion model of rice nitrogen content, and the specific conclusions of this study are as follows:

(1) The hyperspectral range from $400 \mathrm{~nm}$ to $1000 \mathrm{~nm}$ was dimensionally reduced by DWMD, and finally the continuous hyperspectral reflectance information was dimensionally reduced to sixteen discrete hyperspectral features for subsequent inversion modelling of rice nitrogen content.

(2) In the models adopted in this study, BAS-ELM has the highest accuracy, where the R2 of training data, the R2 of test data, the RMSE of training data, and the RMSE of test data is were $0.864,0.863,0.247,0.254$.

\section{Acknowledgments}

This work was supported by Youth Science and Technology Talents Project of the Liaoning Provincial Department of Education (NLSNQN201903).

\section{[References]}

[1] Li B L, Ti C P, Yan X Y. Estimating rice paddy areas in China using multi-temporal cloud-free normalized difference vegetation index (NDVI) imagery based on change detection. Pedosphere, 2020; 30(6): 734-46. doi:10.1016/s1002-0160(17)60405-3

[2] Wang Z H, Skidmore A K, Darvishzadeh R, Wang T J. Mapping forest canopy nitrogen content by inversion of coupled leaf-canopy radiative transfer models from airborne hyperspectral imagery. Agr Forest Meteorol, 2018; 253: 247-60. doi:10.1016/j.agrformet.2018.02.010

[3] Yu F H, Xu T Y, Cao Y L, Yang G J, Du W, Wang S. Models for estimating the leaf NDVI of japonica rice on a canopy scale by combining canopy NDVI and multisource environmental data in Northeast China. Int J Agr Biol Eng, 2016; 9(5): 132-42. doi:10.3965/j.ijabe.20160905.2266

[4] Ata-Ul-Karim S T, Liu X J, Lu Z Z, Zheng H B, Cao W X, Zhu Y. Estimation of nitrogen fertilizer requirement for rice crop using critical nitrogen dilution curve. Field Crop Res, 2017; 201: 32-40. doi: 10.1016/j.fcr.2016.10.009

[5] Tan K Z, Wang S W, Song Y Z, Liu Y, Gong Z P. Estimating nitrogen status of rice canopy using hyperspectral reflectance combined with BPSO-SVR in cold region. Chemometrics Intell Lab Syst, 2018; 172: 68-79. doi: 10.1016/j.chemolab.2017.11.014

[6] Dong Y J, Yuan J, Zhang G B, Ma J, Hilario P, Liu X J, et al. Optimization of nitrogen fertilizer rate under integrated rice management in a hilly area of Southwest China. Pedosphere, 2020; 30(6): 759-68. doi: 10.1016/ s1002-0160(20)60036-4

[7] Dong Y J, Zeng F W, Yuan J, Zhang G B, Chen Y X, Liu X J, et al. Integrated rice management simultaneously improves rice yield and nitrogen use efficiency in various paddy fields. Pedosphere, 2020; 30(6): 863-73. doi: 10.1016/s1002-0160(20)60042-x

[8] Loozen Y, Karssenberg D, de Jong S M, Wang S Q, van Dijk J, Wassen M J, et al. Exploring the use of vegetation indices to sense canopy nitrogen to phosphorous ratio in grasses. International Journal of Applied Earth Observation and Geoinformation, 2019; 75: 1-14.

[9] Fang H L, Baret F, Plummer S, Schaepman-Strub G. An Overview of Global Leaf Area Index (LAI): Methods, Products, Validation, and Applications. Rev Geophys, 2019; 57(3): 739-99. doi: 10.1029/2018rg000608

[10] Corti M, Cavalli D, Cabassi G, Gallina P M, Bechini L. Does remote and proximal optical sensing successfully estimate maize variables? A review. European Journal of Agronomy, 2018; 99: 37-50. doi: 10.1016/ j.eja.2018.06.008

[11] Ata-Ul-Karim S T, Cao Q, Zhu Y, Tang L, Rehmani MIA, Cao W X. Non-destructive Assessment of Plant Nitrogen Parameters Using Leaf Chlorophyll Measurements in Rice. Frontiers in Plant Science, 2016; 7. doi: $10.3389 /$ fpls.2016.01829

[12] Zhang K, Liu XJ, Ata-Ul-Karim S T, Lu J S, Krienke B, Li S Y, et al. Development of Chlorophyll-Meter-Index-Based Dynamic Models for Evaluation of High-Yield Japonica Rice Production in Yangtze River Reaches. Agronomy-Basel, 2019; 9(2). doi:10.3390/agronomy9020106

[13] Zheng H B, Cheng T, Li D, Yao X, Tian Y C, Cao W X, et al. Combining Unmanned Aerial Vehicle (UAV)-Based Multispectral Imagery and Ground-Based Hyperspectral Data for Plant Nitrogen Concentration Estimation in Rice. Frontiers in Plant Science, 2018; 9. doi: 10.3389/ fpls.2018.00936

[14] Li S Y, Ding X Z, Kuang Q L, Ata-Ul-Karim S T, Cheng T, Liu X J, et al Potential of UAV-Based Active Sensing for Monitoring Rice Leaf Nitrogen Status. Frontiers in Plant Science, 2018; 9: 14. doi: 10.3389/ fpls.2018.01834

[15] Lussem U, Bolten A, Menne J, Gnyp M L, Schellberg J, Bareth G. Estimating biomass in temperate grassland with high resolution canopy surface models from UAV-based RGB images and vegetation indices. J Appl Remote Sens, 2019; 13(3): 26. doi: 10.1117/1.Jrs.13.034525

[16] He J Y, Zhang X B, Guo W T, Pan Y Y, Yao X, Cheng T, et al. Estimation of Vertical Leaf Nitrogen Distribution Within a Rice Canopy Based on Hyperspectral Data. Frontiers in Plant Science, 2020; 10. doi: ARTN 1802.10.3389/fpls.2019.01802

[17] Berger K, Verrelst J, Feret J B, Wang Z H, Wocher M, Strathmann M, et al. Crop nitrogen monitoring: Recent progress and principal developments in the context of imaging spectroscopy missions. Remote Sensing of Environment, 2020; 242: 18. doi: 10.1016/j.rse.2020.111758

[18] Ge X Y, Wang J Z, Ding J L, Cao X Y, Zhang Z P, Liu J, et al. Combining UAV-based hyperspectral imagery and machine learning algorithms for soil moisture content monitoring. Peer J, 2019; 7: 27. doi: 10.7717/peerj.6926

[19] Din M, Ming J, Hussain S, Ata-Ul-Karim S T, Rashid M, Tahir M N, et al. Estimation of Dynamic Canopy Variables Using Hyperspectral Derived Vegetation Indices Under Varying N Rates at Diverse Phenological Stages of Rice. Frontiers in Plant Science, 2019; 9. doi: 10.3389/fpls.2018.01883

[20] Jay S, Baret F, Dutartre D, Malatesta G, Heno S, Comar A, et al. Exploiting the centimeter resolution of UAV multispectral imagery to improve remote-sensing estimates of canopy structure and biochemistry in sugar beet crops. Remote Sensing of Environment, 2019; 231: 17. doi: 10.1016/ j.rse.2018.09.011

[21] Camino C, González-Dugo V, Hernández P, Sillero J C, Zarco - Tejada PJ. Improved nitrogen retrievals with airborne-derived fluorescence and plant traits quantified from VNIR-SWIR hyperspectral imagery in the context of precision agriculture. International Journal of Applied Earth Observation and Geoinformation, 2018; 70: 105-17. doi: 10.1016/j.jag.2018.04.013

[22] Masemola C, Cho M A. Estimating leaf nitrogen concentration from similarities in fresh and dry leaf spectral bands using a model population analysis framework. Int J Remote Sens, 2019; 40(17): 6841-60. doi: 10.1080/01431161.2019.1597300

[23] Klem K, Zahora J, Zemek F, Trunda P, Tuma I, Novotna K, et al Interactive effects of water deficit and nitrogen nutrition on winter wheat. Remote sensing methods for their detection. Agric Water Manage, 2018; 210: 171-84. doi: 10.1016/j.agwat.2018.08.004

[24] Sothe C, Dalponte M, de Almeida C M, Schimalski M B, Lima C L, Liesenberg V, et al. Tree Species Classification in a Highly Diverse Subtropical Forest Integrating UAV-Based Photogrammetric Point Cloud and Hyperspectral Data. Remote Sens-Basel, 2019; 11(11): 24 . doi: $10.3390 /$ rs 11111338

[25] Zheng H B, Cheng T, Li D, Yao X, Tian Y C, Cao W X, et al. Combining Unmanned Aerial Vehicle (UAV)-Based Multispectral Imagery and Ground-Based Hyperspectral Data for Plant Nitrogen Concentration Estimation in Rice. Frontiers in Plant Science, 2018; 9: 13. doi: 10.3389/fpls.2018.00936

[26] Diacono M, Rubino P, Montemurro F. Precision nitrogen management of wheat. A review. Agron Sustain Dev, 2013; 33(1): 219-41. doi: 10.1007/ s13593-012-0111-z

[27] Reshma S, Veni S, George J E, Ieee. Hyperspectral Crop Classification Using Fusion of Spectral, Spatial Features and Vegetation Indices: Approach to the Big Data Challenge. New York: Ieee; 2017, 380-6 p.

[28] Du W, Xu T Y, Yu F H, Chen C L. Measurement of nitrogen content in rice by inversion of hyperspectral reflectance data from an unmanned aerial vehicle. Cienc Rural, 2018; 48(6): 10 . doi: 10.1590/ 0103-8478cr20180008 